\title{
Modal Decay in the Australia-Antarctic Basin
}

\author{
WILBERT WEIJER \\ Los Alamos National Laboratory, Los Alamos, New Mexico \\ SARAH T. GILLE \\ Scripps Institution of Oceanography, La Jolla, California \\ FRÉDÉRIC VIVIER \\ LOCEAN IPSL, Paris, France
}

(Manuscript received 5 January 2009, in final form 11 June 2009)

\begin{abstract}
The barotropic intraseasonal variability in the Australia-Antarctic Basin (AAB) is studied in terms of the excitation and decay of topographically trapped barotropic modes. The main objective is to reconcile two widely differing estimates of the decay rate of sea surface height (SSH) anomalies in the AAB that are assumed to be related to barotropic modes. First, an empirical orthogonal function (EOF) analysis is applied to almost 15 years of altimeter data. The analysis suggests that several modes are involved in the variability of the $\mathrm{AAB}$, each related to distinct areas with (almost) closed contours of potential vorticity. Second, the dominant normal modes of the AAB are determined in a barotropic shallow-water (SW) model. These stationary modes are confined by the closed contours of potential vorticity that surround the eastern $\mathrm{AAB}$, and the crest of the Southeast Indian Ridge. For reasonable values of horizontal eddy viscosity and bottom friction, their decay time scale is on the order of several weeks. Third, the SW model is forced with realistic winds and integrated for several years. Projection of the modal velocity patterns onto the output fields shows that the barotropic modes are indeed excited in the model, and that they decay slowly on the frictional $\mathcal{O}(3$ weeks $)$ time scale. However, the SSH anomalies in the modal areas display rapid $\mathcal{O}(4$ days $)$ decay. Additional analysis shows that this rapid decay reflects the adjustment of unbalanced flow components through the emission of Rossby waves. Resonant excitation of the dominant free modes accounts for about $20 \%$ of the SSH variability in the forced-model run. Other mechanisms are suggested to explain the region of high $\mathrm{SSH}$ variability in the AAB.
\end{abstract}

\section{Introduction}

Studies of altimeter data and numerical models have identified several areas in the world's oceans that are characterized by excessively high levels of intraseasonal variability (Chao and Fu 1995; Fu and Smith 1996; Fukumori et al. 1998; Ponte and Gaspar 1999; Webb and De Cuevas 2002a,b, 2003; Fu 2003; Vivier et al. 2005; Weijer and Gille 2005). Several of these areas coincide with pronounced abyssal plains, such as the Bellingshausen Basin, the Argentine Basin, and the Australia-Antarctic Basin. These basins are character-

Corresponding author address: Wilbert Weijer, Los Alamos National Laboratory, CCS-2, MS B296, Los Alamos, NM 87545. E-mail: wilbert@lanl.gov ized by closed, or almost closed, contours of potential vorticity $f / H$ (where $H$ is the local water depth and $f$ is the Coriolis parameter). This has led to the suggestion that the variability is caused by the excitation of topographically trapped barotropic wave modes (e.g., Fukumori et al. 1998; Webb and De Cuevas 2002a,b).

Webb and De Cuevas (2002a, hereafter WD02) studied the variability in the Australia-Antarctic Basin (AAB) in considerable detail. They applied an empirical orthogonal function (EOF) analysis to the sea surface height (SSH) fields from their eddy-admitting ocean model Ocean Circulation and Climate Advanced Modelling Project (OCCAM) and found that the time series of the dominant EOF correlated well with local measures of Ekman pumping. A coherence analysis between the forcing and EOF time series yielded a decay time scale of the 
EOF of only 3.45 days. Fu (2003, hereafter F03) addressed the high variability in this region from an observational point of view. Coherence between scatterometer-derived wind stress curl and altimeter-based sea level anomaly at a particular location in the $\mathrm{AAB}$ appeared to suggest a decay time scale of no less than 30 days.

The discrepancy between these estimates is considerable and has large consequences for our understanding of the decay of barotropic motions and its importance for the energy balance of the ocean. In this paper we will study the role that topographically trapped barotropic modes play in the intraseasonal variability of the AAB. We will analyze altimeter observations to study the statistical modes of variability in the $\mathrm{AAB}$, perform normalmode analysis to determine the dominant barotropic modes, and perform transient integrations with a shallowwater (SW) model to study the dynamics of the enhanced intraseasonal variability. The main conclusions are that (i) barotropic modes indeed explain a part of the observed $\mathrm{SSH}$ variance; (ii) their decay is governed by frictional spindown; and (iii) the apparent rapid decay of SSH reflects the transient adjustment of unbalanced flow components, rather than the decay of the actual free modes.

\section{Linking statistical modes of variability to normal modes}

\section{a. Statistical analysis: The dominant EOFs of the $A A B$}

In this section the variability in the $\mathrm{AAB}$ is studied using a statistical (EOF) analysis of SSH observations. It extends the work of F03 by using longer altimetric time series and by considering multiple modes of variability.

The SSH dataset used here consists of merged Ocean Topography Experiment (TOPEX)/Poseidon (T/P) and European Remote Sensing Satellite (ERS) altimeter observations, mapped onto a $1 / 3^{\circ}$ Mercator grid, and provided by Archiving, Validation, and Interpretation of Satellite Oceanographic data (AVISO; Ducet et al. 2000). The data have been box averaged to a $4^{\circ}$ spatial resolution to reduce the signature of eddies. Grid points contaminated by sea ice are discarded. The data are weekly values from October 1992 to May 2007. A Parks-McClellan filter (Parks and McClellan 1972) of order 40 (with a transition zone from $1 / 187$ to $1 / 80 \mathrm{cpd}$ ) has been applied to remove the seasonal cycle and low-frequency variability.

The three dominant EOFs of the high-pass-filtered weekly SSH data account for roughly half of the variance of the filtered data (Fig. 1, left panels). EOF 1 is similar to the empirical mode found by F03, and also resembles the EOF found by WD02 in their model. Its monopolar pattern captures variance in the entire area east of Kerguelen Plateau, including the eastern AAB (also sometimes referred to as the Wilkes Abyssal Plain) and Southeast Indian Ridge. The core of maximum variance is a zonal band between $100^{\circ}$ and $120^{\circ} \mathrm{E}$ at $53^{\circ} \mathrm{S}$, with an additional maximum at $57^{\circ} \mathrm{S}, 93^{\circ} \mathrm{E}$. EOF 2 has a maximum in the eastern $\mathrm{AAB}$, and a minimum in the western part, off the Kerguelen Plateau (also known as Kerguelen Abyssal Plain). The expression of EOF 3 is concentrated on top of the Southeast Indian Ridge.

Richman (1986) argued that EOFs have several disadvantages that often impede the interpretation of EOFs in terms of physical modes. One of those problems is a strong dependence on domain shape that is often reflected in a predictable monopole-dipole-tripole morphological sequence of the dominant EOFs. He showed that rotation of the EOFs can yield patterns that are in closer agreement with the physical modes that are responsible for the variability. Since the dominant EOFs in our analysis might suffer from this domain shape dependence, a varimax rotation was applied to the first 10 renormalized EOFs. The first three rotated EOFs (rEOFs) are shown in Fig. 1 (the right panels). The rEOFs suggest that the actual patterns of variance might be more localized than suggested by the EOFs, namely, over the Southeast Indian Ridge (rEOF 1), in the western $\mathrm{AAB}$ (rEOF 2), and in the eastern AAB (rEOF 3). In fact, this localization appears to coincide with areas with (almost) closed contours of $f / H$.

This analysis suggests that the dominant EOFs capture the variance of several modes of variability. Rotation of the EOFs localizes and focuses the patterns of variability, and places them over areas with closed contours of $f / H$. This lends support to the suggestion by WD02 and F03 that the SSH variability may be caused by topographically trapped barotropic modes.

\section{b. Model analysis: The dominant normal modes of the $A A B$}

To test whether modes exist that are consistent with the observations of the previous section, we perform a normal-mode analysis of the Southern Ocean in a barotropic shallow-water context (Weijer et al. 2007b). Details of the model formulation and solution method can be found in the appendix. The model domain ranges from $-70^{\circ}$ to $0^{\circ}$ latitude and from $45^{\circ}$ to $185^{\circ}$ east longitude. The numerical grid consists of $210 \times 210$ grid points, which yields a spatial resolution of $2 / 3^{\circ} \times 1 / 3^{\circ}$. Bathymetry is based on the global 2-min gridded elevations/bathymetry for the world dataset (ETOPO-2), box averaged onto our model grid and smoothed by applying a filter to remove the smallest length scales (Fig. 2). Depths smaller than $300 \mathrm{~m}$ (the continental shelf) are set to zero. Horizontal eddy viscosity is parameterized through a Laplacian formulation with 

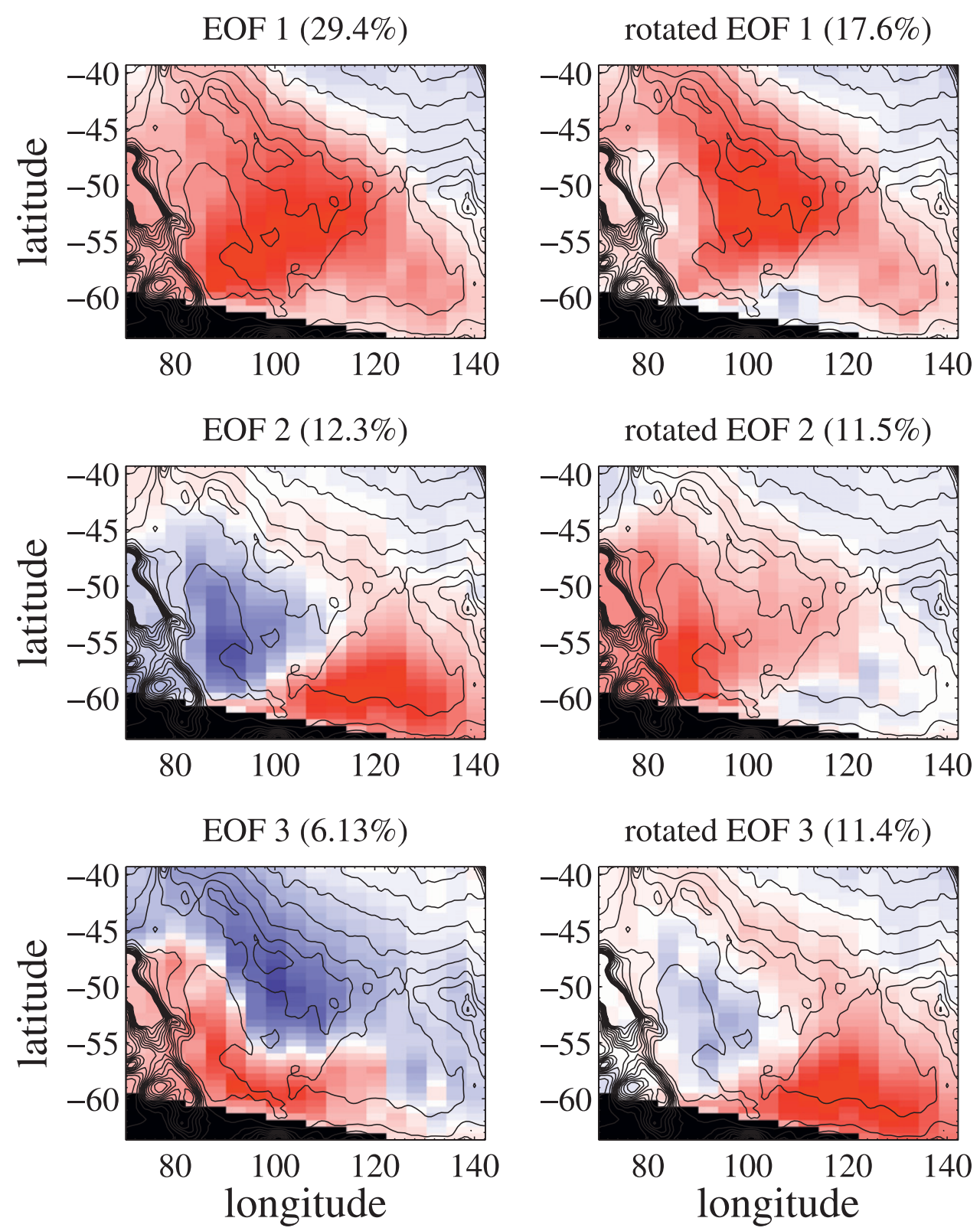

FIG. 1. (left) Unrotated and (right) rotated EOFs of altimeter data in the AAB. Together, they account for about half of the variance. The spatial patterns are regridded onto a $4^{\circ} \times 0.6^{\circ}$ grid for visualization purposes only. Amplitudes are undetermined, but the fields are scaled with their maximum (absolute) values; color ranges from blue (-1) through white (0) to red (1). Contours are isolines of the base- 10 logarithm of $f / H$, plotted for the interval $[-9.0,-7.0]$ with step 0.5 .

coefficient $A_{h}$, while bottom friction is represented through a linear drag with coefficient $r$.

The barotropic modes are determined by performing a normal-mode analysis on the unforced, motionless solution of this model. The two dominant normal modes are shown in Fig. 3. Their spatial patterns will be indicated by $\mathbf{m}_{i}(\mathbf{x})=\left[m_{i}^{u}(\mathbf{x}), m_{i}^{v}(\mathbf{x}), m_{i}^{\eta}(\mathbf{x})\right]$, where $\mathbf{x}=(\lambda, \phi)$ are the coordinates in longitude $\lambda$ and latitude $\phi$, and $m_{i}^{u}, m_{i}^{v}$, and $m_{i}^{\eta}$ denote the spatial patterns of $u, v$, and $\eta$, respectively, associated with modes $i=1,2$. In contrast to the modes in the Argentine Basin (Weijer et al. 2007b) and the Mascarene Basin (Weijer 2008), these modes are stationary and do not represent oscillatory motion. Technically, the normal modes as well as their eigenvalues are realvalued. Once excited, they decay with a rate governed by their associated eigenvalues (see the appendix).

Mode 1 represents a triangular-shaped mode in the eastern AAB. This mode is clearly confined to the area 


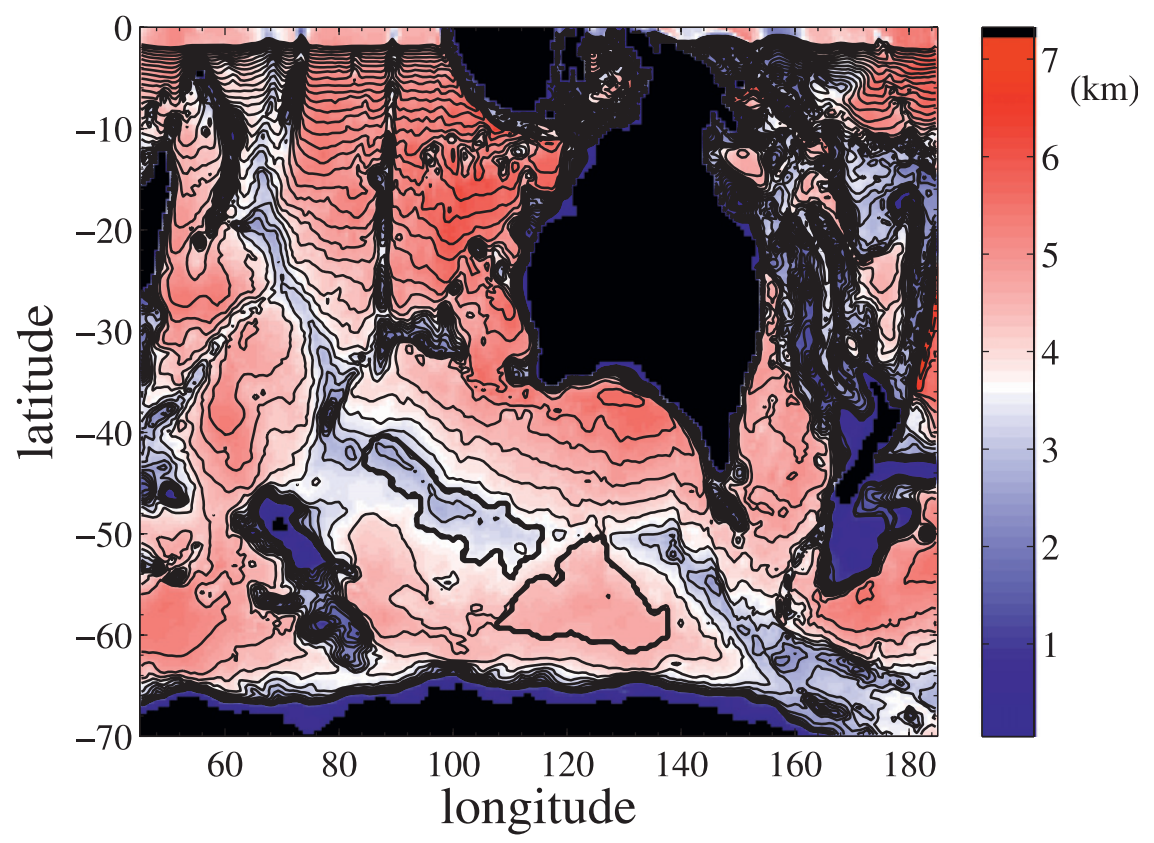

FIG. 2. Bathymetry of the computational domain. Contours show the base-10 logarithm of $f / H$, plotted for the interval $[-9.0,-7.0]$ with step 0.5 . The thick black lines define the modal areas $M_{1}$ and $M_{2}$ and are defined by the isolines $f / H=2.82 \times 10^{-8} \mathrm{~m}^{-1} \mathrm{~s}^{-1}$ and $3.16 \times$ $10^{-8} \mathrm{~m}^{-1} \mathrm{~s}^{-1}$ that surround the abyssal plain of the eastern AAB and the crest of the Southeast Indian Ridge, respectively.

of closed contours of $f / H$ that outlines the abyssal plain. The last contour that is still continuous has the approximate value of $f / H=2.82 \times 10^{-8} \mathrm{~m}^{-1} \mathrm{~s}^{-1}$, and it outlines an area that will be referred to as $M_{1}$. The velocity field associated with this pattern of $\eta$ encircles the abyssal plain. The spatial pattern shows a close resemblance to the statistical pattern of SSH variability rEOF 3 (Fig. 1). Mode 2 is also trapped by closed contours of $f / H$, here surrounding the crest of the Southeast Indian Ridge. The isoline $3.16 \times 10^{-8} \mathrm{~m}^{-1} \mathrm{~s}^{-1}$ is the last contour that is still continuous and defines the modal area $M_{2}$. The modal velocity features opposite flow directions on either flank of the ridge. Its pattern resembles rEOF 1 (Fig. 1).

The decay rate of the modes depends strongly on the frictional parameters. Figure 4 shows the result of a sensitivity study in the parameter space spanned by the coefficients for bottom friction $r$ and horizontal viscosity $A_{h}$. For frictional values $A_{h}=3 \times 10^{3} \mathrm{~m}^{2} \mathrm{~s}^{-1}$ and $r=2.0 \times$ $10^{-7} \mathrm{~s}^{-1}$, the decay rates are on the order of 3 weeks. This is consistent with the conventional view of frictional spindown (e.g., F03).

\section{c. Estimating the decay time scale}

Both WD02 and F03 have shown that the variability in the $\mathrm{AAB}$ is correlated to local measures of wind stress curl, and they used the coherence phase between relevant forcing and response time series to estimate the decay time scale of the response. Here we will make another estimate of the decay time scale by projecting the spatial patterns of the dynamically consistent modes onto SSH data. The idea is that this will give us time series that represent the amplitude of the barotropic modes (although later we will show that this is not necessarily a valid assumption). Weijer et al. (2007a) used this technique successfully to demonstrate the presence of multiple barotropic normal modes in the Argentine Basin.

We assume that the actual evolution of the dynamical fields $\mathbf{S}=\left(S^{u}, S^{v}, S^{\eta}\right)$ consists of noise $\mathbf{Z}=\left(Z^{u}, Z^{v}, Z^{\eta}\right)$ and a sum $\mathbf{S}_{m}$ of modal responses with spatial patterns $\mathbf{m}_{i}=\left(m_{i}^{u}, m_{i}^{v}, m_{i}^{\eta}\right)$ and amplitude time series $\gamma_{i}$ :

$$
\mathbf{S}(\mathbf{x}, t)=\mathbf{S}_{m}(\mathbf{x}, t)+\mathbf{Z}(\mathbf{x}, t)
$$

with

$$
\mathbf{S}_{m}(\mathbf{x}, t)=\sum_{i} \gamma_{i}(t) \mathbf{m}_{i}(\mathbf{x})
$$

where for our purpose $i \in[1,2]$. Multiplying Eq. (1) by each of the modal patterns $\mathbf{m}_{k}$ and integrating over the domain $D$ yields 

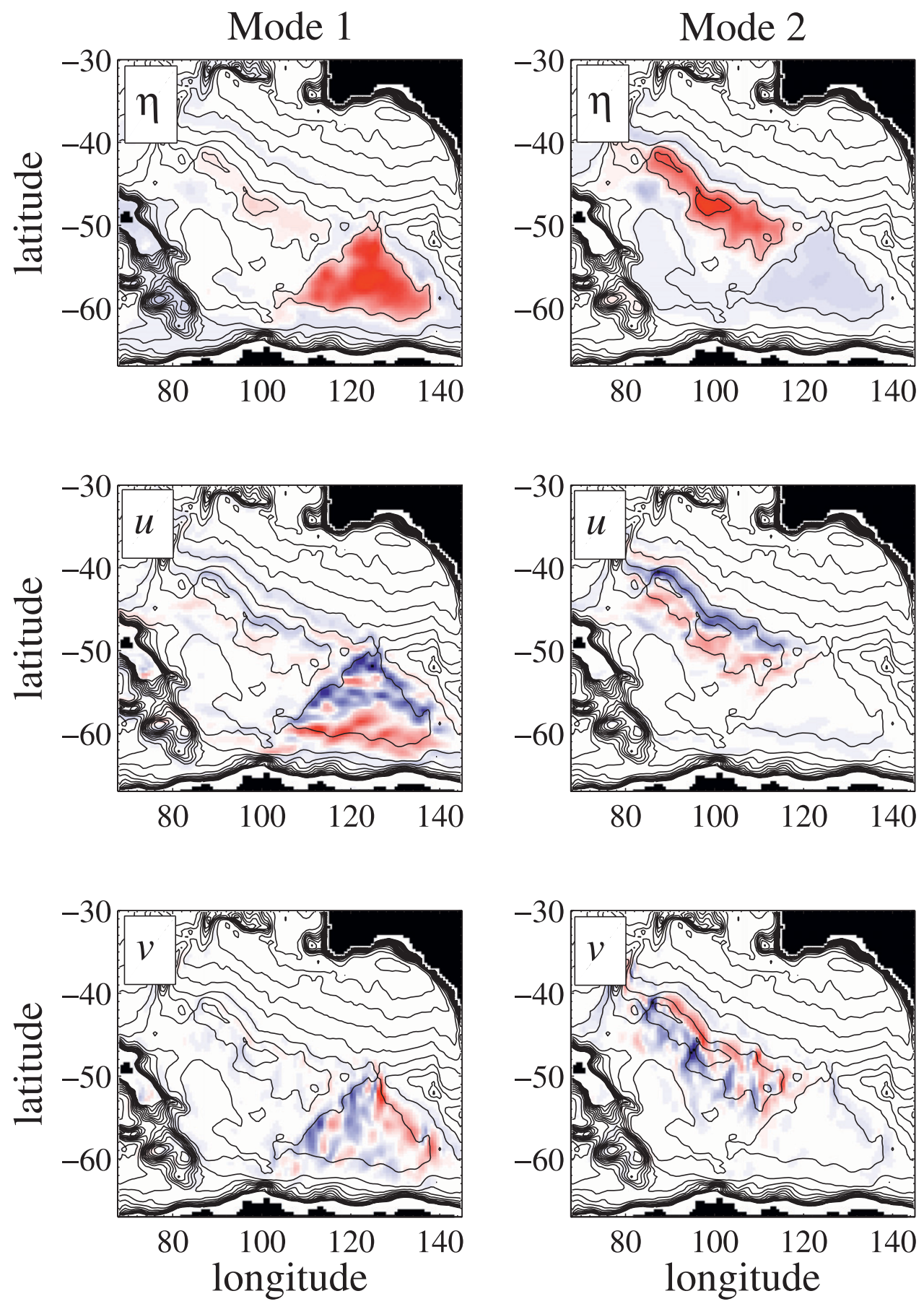

FIG. 3. Normal modes of the AAB. (top) Sea surface elevation $\eta$, (middle) zonal velocity $u$, and (bottom) meridional velocity $v$ for mode (left) 1 and (right) 2 . Friction coefficients are $A_{h}=3 \times$ $10^{3} \mathrm{~m}^{2} \mathrm{~s}^{-1}$ for horizontal eddy viscosity, and $r=2.0 \times 10^{-7} \mathrm{~s}^{-1}$ for bottom friction. The decay time scales for these values of friction are 19.7 days for mode 1 and 18.4 days for mode 2. Amplitudes are undetermined, but mutually consistent. Color scale ranges from $-\max |x|$ (blue) to $\max |x|$ (red) through zero (white). In particular, when SSH elevations are scaled to a maximum amplitude of $1 \mathrm{~m}$, the range in zonal and meridional velocities is $[-0.44,0.42]$ and $[-0.44,0.64] \mathrm{m} \mathrm{s}^{-1}$ for mode 1 , and $[-0.85,0.39]$ and $[-0.53,0.42] \mathrm{m} \mathrm{s}^{-1}$ for mode 2. Contours show base-10 $\log$ of $f / H$. 


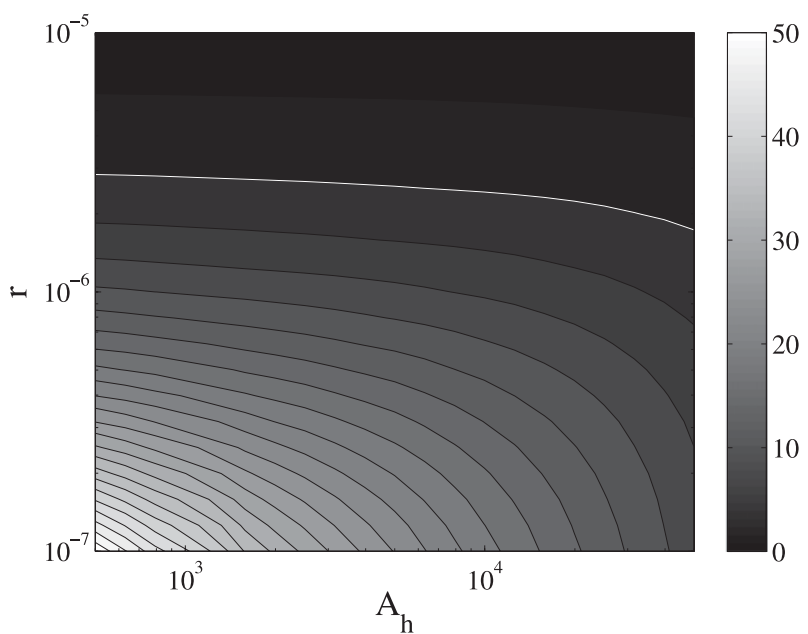

FIG. 4. Decay time scale of mode 2 (in days) as function of bottom-friction coefficient $r$ and horizontal viscosity $A_{h}$. White line indicates 4-day decay time scale.

$$
\int_{D} \mathbf{m}_{k} \cdot \mathbf{S} d A=\sum_{i=1}^{2} \gamma_{i}(t) \int_{D} \mathbf{m}_{k} \cdot \mathbf{m}_{i} d A+\int_{D} \mathbf{m}_{k} \cdot \mathbf{Z} d A
$$

The last integral in Eq. (3) vanishes since it is assumed that the noise is spatially uncorrelated to the eigenmodes. Also, modes 1 and 2 do not overlap so their spatial patterns are orthogonal. This equation suggests that $\gamma_{i}$ can be estimated from each of the three variables independently, according to

$$
\gamma_{i}^{s}(t)=\frac{\int_{D} m_{i}^{s} S^{s} d A}{\int_{D} m_{i}^{s} m_{i}^{s} d A},
$$

where $s=u, v$, or $\eta$.

Here this procedure is applied to the AVISO altimeter data described in section 2a. To generate the response time series, the SSH fields of the normal modes $\left(m_{i}^{\eta}\right.$; Fig. 3, top panels) are first interpolated onto the Mercator grid of the gridded altimeter data.

To construct the forcing time series, the same projection technique is applied to the blended wind product described by Milliff et al. (2004), which consists of Quick Scatterometer (QuikSCAT) data blended with National Centers for Environmental Prediction (NCEP) reanalyses. The data are provided on a $0.5^{\circ}$ grid at 6-hourly intervals for the time period from July 1999 to June 2006. Leap days are absent but are filled in by linear interpolation. The spatial patterns of the modes are projected on the wind stress curl fields according to
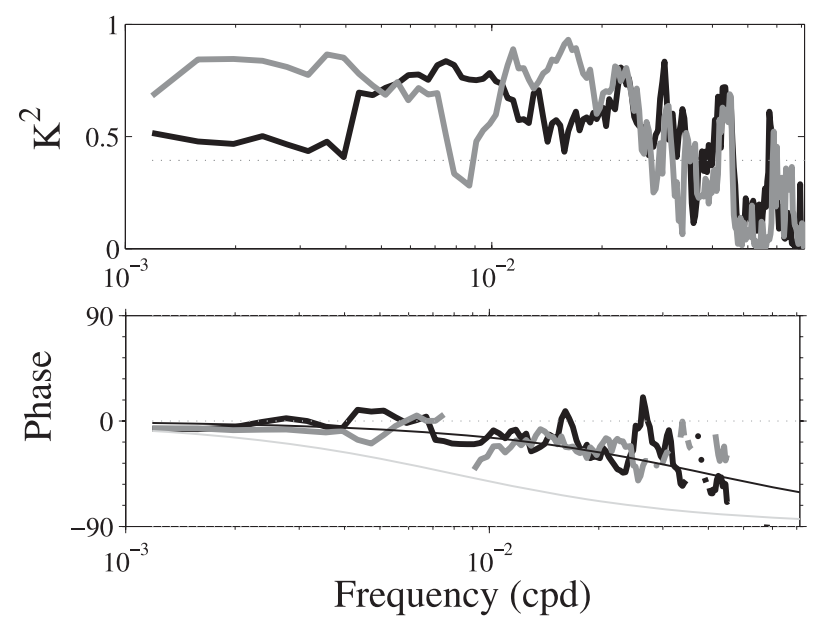

FIG. 5. The (squared) coherence and coherence phase between amplitude and forcing time series for mode 1 (black lines) and mode 2 (gray lines). The dotted line in coherence panel shows $95 \%$ significance level. Thin lines indicate the phase lag between two time series that satisfy Eq. (5), with a fixed decay time $\tau$ of 4 (black) and 20 (gray) days, respectively. The $95 \%$ confidence limits of the phase spectra (not shown) were determined according to the expression given by Hannan (1970). The corresponding error in the time lag was found to be smaller than 3 days for all frequencies, except for the range $f<0.004 \mathrm{cpd}$ for mode 1 and $f<0.002 \mathrm{cpd}$ for mode 2, where the uncertainties are higher.

Eq. (4) to construct the forcing time series. A ParksMcClellan filter of order 40 is applied to retain periods exceeding 7 days. The resulting time series are then sampled at $\mathrm{SSH}$ time steps.

Figure 5 shows the (squared) coherence and phase between the projection amplitude time series and the wind stress curl time series. For both modes the coherence is significant for time scales exceeding a month. This supports the conclusion of WD02 and F03 that the SSH variability is indeed excited by the wind stress curl.

The coherence phase is very small for both modes. The thin lines in the phase panel denote the phase lag between forcing and response for decay time scales $\tau$ of 4 days (black) and 20 days (gray), if we suppose the response $\Psi$ of the system to be related to the forcing $T$ through the simple forcing-response model (Weijer and Gille 2005):

$$
\frac{d \Psi}{d t}=T-\frac{\Psi}{\tau}
$$

Since the system is linear, Eq. (5) should hold for every frequency component individually, so that in Fourier space we have

$$
\left(2 \pi i f+\frac{1}{\tau}\right) \Psi^{*}=T^{*}
$$




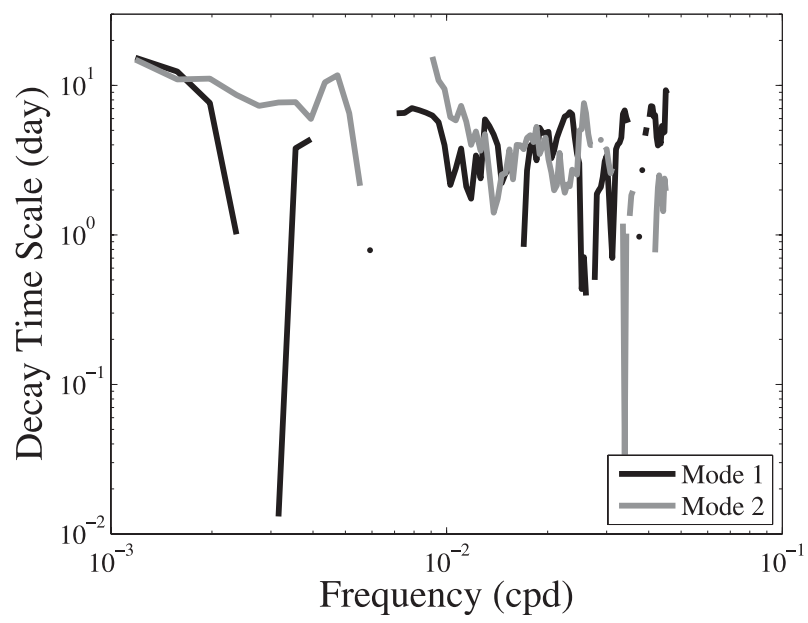

FIG. 6. Decay time scale as estimated from Eq. (10).

where $\tau$ is allowed to depend on frequency. Multiplying the equation with the complex conjugate of $\Psi^{*}, \tilde{\Psi}^{*}$, we get

$$
\left(2 \pi i f+\frac{1}{\tau}\right) \Psi^{*} \tilde{\Psi}^{*}=T^{*} \tilde{\Psi}^{*}
$$

where we recognize the cross-spectrum $T^{*} \tilde{\Psi}^{*}$. The cross spectrum is complex-valued, and can be decomposed in a cospectrum $(\Lambda)$ and quadrature spectrum $(\Gamma)$, so that

$$
\left(2 \pi i f+\frac{1}{\tau}\right)\left|\Psi^{*}\right|^{2}=\Lambda+i \Gamma
$$

The phase spectrum $\varphi(f)$ can now be written as

$$
\tan \varphi=\frac{\Gamma}{\Lambda}=2 \pi f \tau
$$

So for two given time series, $\tau$ can be estimated from

$$
\tau=\frac{\tan \varphi}{2 \pi f}
$$

Alternatively, if the relation between $\Psi$ and $F$ is quantitatively well known, $\tau$ can be estimated directly from the cospectrum:

$$
\tau=\frac{\left|\Psi^{*}\right|^{2}}{\Lambda}
$$

The decay time scales determined according to Eq. (10) are plotted in Fig. 6. Clearly, the values are shorter than a week. Mean values are 5.3 days (4.2 days) for mode 1 (mode 2). These values correspond well with the values found by WD02, but are at odds with the decay time
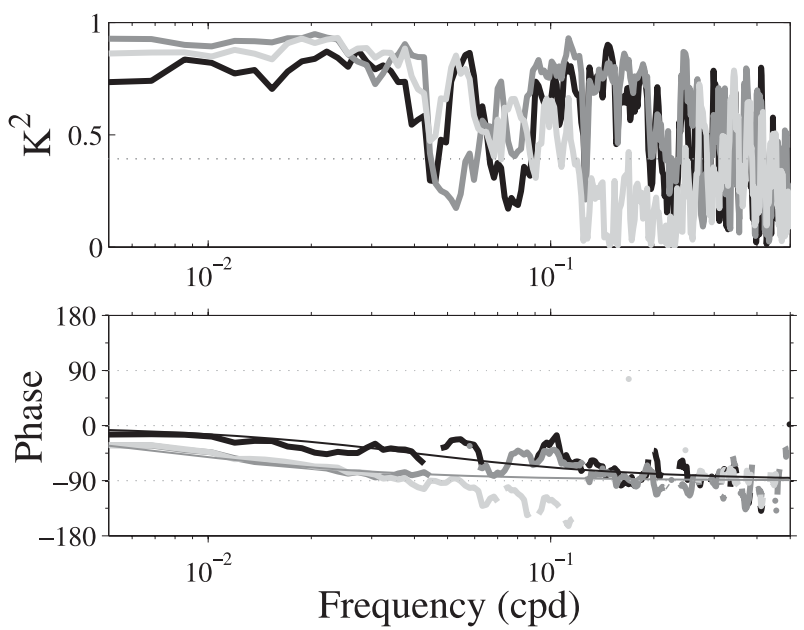

FIG. 7. The (squared) coherence and coherence phase between amplitude and forcing time series for mode 1. Black, dark gray, and light gray denote the coherence between area-averaged wind stress curl and amplitude time series $\gamma_{1}^{\eta}, \gamma_{1}^{u}$, and $\gamma_{1}^{v}$, respectively. The dotted line in the coherence panel shows $95 \%$ significance level. Thin black and gray lines are consistent with decay time scales of 4 and 20 days, respectively.

scale inferred from the normal-mode analysis, as well as with estimates by F03. A close inspection of the data used by F03 suggests that a bias in the timing of his wind stress curl data may have been the cause for the apparent long decay time scales.

\section{Mechanisms of decay: Numerical modeling \\ a. Estimating the decay time scale in the shallow-water model}

The discrepancy between the expected decay rate of the modes and the estimate based on SSH observations is puzzling. To study the modal decay in more detail, we integrated the SW model for 840 days, starting 1 August 1999. The model was forced by the Milliff et al. (2004) wind stress data described in section 2c. Nonlinear advection terms are ignored in these calculations. To reduce the impact of low-frequency large-scale circulations in this unrealistically bounded domain, the forcing was filtered to remove the annual and semiannual cycles and detrended. We used a Crank-Nicolson scheme with a 15-min time step. Daily averages of the dynamical fields $\eta, u$, and $v$ are saved.

To check whether SSH in the model experiences a similar rapid decay as in observations, we followed the same procedure as in the former section, and projected the modal patterns of $\eta$ onto the daily averaged SSH fields. The coherence between the time series $\gamma_{1}^{\eta}$ [Eq. (4)] and the wind stress curl averaged over modal area 1 is plotted in Fig. 7 (black line). For the model, as for the 
data, the phase spectrum clearly shows that the relation between forcing and SSH response is consistent with a rapid decay on the order of 4 days.

However, the model integration provides us with the explicit velocity fields as well. This enables us to apply the projection technique to the zonal and meridional velocity components (middle and bottom panels of Fig. 3), and to construct time series $\gamma_{i}^{u}$ and $\gamma_{i}^{v}$ according to Eq. (4). The coherence of the resulting projection time series with wind stress curl is plotted for mode 1 as the dark gray and light gray lines in Fig. 7. There is again a significant coherence between the wind stress curl forcing in $M_{1}$ and the amplitude time series $\gamma_{1}^{u}$ and $\gamma_{1}^{v}$ of the zonal and meridional velocity components. But interestingly enough, the phase spectra are now consistent with a slow decay time scale on the order of 20 days.

The most consistent interpretation of this result is that the amplitude of the actual free modes is reflected by the projection time series $\gamma^{u}$ and $\gamma^{v}$, rather than by the time series $\gamma^{\eta}$. The physical basis for this conclusion is that the slow decay time scale suggested by the statistical analysis of $\gamma^{u}$ and $\gamma^{v}$ is consistent with the decay rate predicted by the normal-mode analysis for the (reasonable) values of the frictional parameters used here. In addition, the premise that the projection coefficients reflect the modal amplitude depends on the assumption that the noise terms $Z^{s}$ in Eq. (3) are orthogonal to the modal patterns $m_{i}^{s}$. The fact that $\gamma^{\eta} \neq \gamma^{u}, \gamma^{v}$ while $\gamma^{u} \sim \gamma^{v}$ suggests that this condition is not satisfied for $\eta$. The close correspondence between $\gamma_{i}^{u}$ and $\gamma_{i}^{v}$ means that noise would have to corrupt the projection time series of the two velocity components in exactly the same way, which is unlikely. Also, the spatial patterns $m_{i}^{u}$ and $m_{i}^{v}$ have values of both signs, making them much more selective than the patterns of $m_{i}^{\eta}$ (see Fig. 3). The latter have nonzero projection on SSH signals that envelop the modal areas $M_{1}$ and $M_{2}$. These results suggest that the time series $\gamma_{i}^{\eta}$ are corrupted by nonzero projections of the modal patterns $m_{i}^{\eta}$ on "noise" with large spatial scales.

\section{b. The vorticity balance}

To take a closer look at the processes leading to modal decay, we consider the balance of vorticity of the depthaveraged flow. The momentum equations are

$$
\frac{d \mathbf{u}}{d t}+f \hat{\mathbf{k}} \times \mathbf{u}=-g \nabla \eta-r \mathbf{u}+\frac{\tau}{\rho_{0} H},
$$

where $d / d t$ indicates the local derivative, since advective processes are negligible (and ignored in the simulations). The sum of bottom friction and horizontal vis- cosity is schematically represented by the Rayleigh term [second term in the right-hand side of Eq. (12)].

The vorticity equation follows by taking the curl of Eq. (12):

$$
\frac{d Z}{d t}+\nabla \times(f \hat{\mathbf{k}} \times \mathbf{u})=-r Z+\nabla \times \frac{\tau}{\rho_{0} H},
$$

where $Z=\nabla \times \mathbf{u}$ is the relative vorticity. We have

$$
\boldsymbol{\nabla} \times(f \hat{\mathbf{k}} \times \mathbf{u})=\frac{f}{H} \nabla \cdot H \mathbf{u}+H \mathbf{u} \cdot \nabla\left(\frac{f}{H}\right)
$$

so that

$$
\frac{d Z}{d t}=-\frac{f}{H} \nabla \cdot H \mathbf{u}-H \mathbf{u} \cdot \nabla\left(\frac{f}{H}\right)-r Z+\nabla \times \frac{\tau}{\rho_{0} H} .
$$

The terms on the right represent vortex stretching (VS), bottom torque $(\mathrm{BT})$, friction $(R)$, and wind stress curl forcing $(F)$, respectively.

The terms in Eq. (15) were evaluated and integrated over the modal areas, indicated by $M_{1}$ and $M_{2}$. Figure 8a shows the spectra of each of these terms integrated over $M_{1}$, while Fig. 8b shows the (squared) coherence and coherence phase between relative vorticity $(Z)$ and the wind stress curl $(F)$, and the (negative of) friction $(-R)$, vortex stretching $(-\mathrm{VS})$, and bottom torque $(-\mathrm{BT})$ terms.

It is clear that the vorticity input by the wind stress is absorbed dominantly by the tendency of relative vorticity, suggesting a spinup of a geostrophic vortex in direct response to the forcing. Indeed, relative vorticity is highly coherent with wind stress curl for the entire frequency range. Their phase difference shows the characteristic relationship of the form $d Z / d t=F-Z / \tau$, where the decay time scale $\tau$ is consistent with 20 days (thin black line in coherence phase plot). The question is what processes are responsible for damping the relative vorticity. A necessary characteristic for a process to really dissipate relative vorticity is that it is coherent and in antiphase with $Z$ (i.e., a phase near zero in Fig. $8 \mathrm{~b}$ where the negative of the terms of the vorticity balance are considered).

The spectral plot shows different balances in three distinct frequency bands. For high frequencies, $f>1 / 12 \mathrm{cpd}$, the VS term accounts for most of the residual $d Z / d t-F$. The term is in quadrature with $Z$ and in phase with $F$, suggesting that the vortex stretching reflects the change in SSH associated with the spinup of a geostrophic vortex: it buffers a fraction of the vorticity input by $F$. It is clear that this term is not able to dissipate vorticity. The 

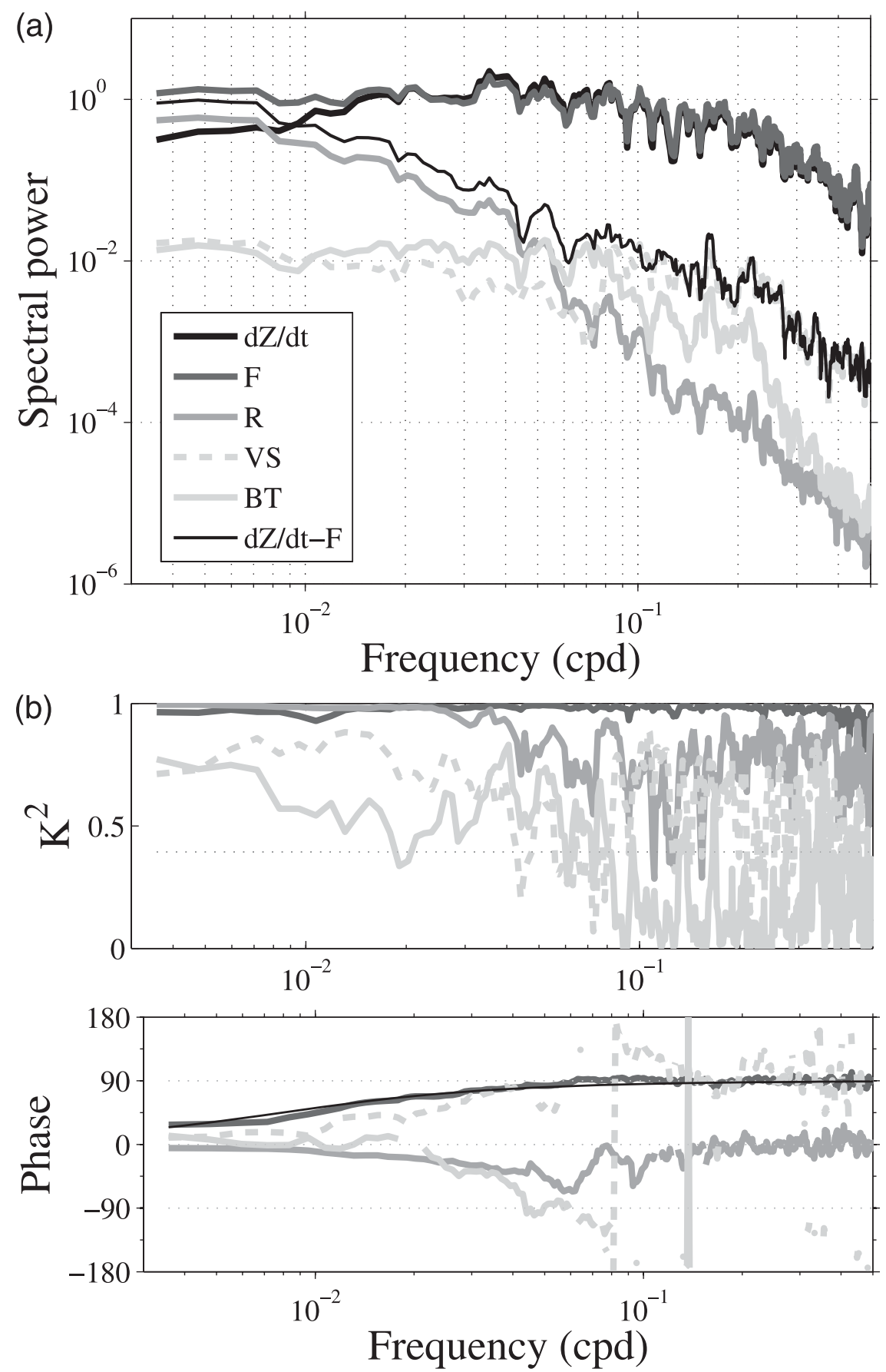

FIG. 8. (a) Power spectra of the terms of the vorticity equation for region $M_{1}$. (b) Coherence of relative vorticity $(Z)$ with the wind stress $\operatorname{curl}(F)$, and with the (negative of) friction $(-R)$, vortex stretching $(-\mathrm{VS})$, and bottom torque $(-\mathrm{BT})$ terms. Thin solid line in phase plot is consistent with a 20-day decay time scale.

bottom torque term - BT (solid light gray) in this frequency range has less power than VS, suggesting that the forced transport is directed mostly along lines of $f / H$. This is consistent with the spinup of a free barotropic mode. In addition, BT is coherent with neither $Z$ nor $F$. The power associated with this term might hence reflect the passage of Rossby waves through the domain. The term with the least power is friction $R$. Nonetheless, it is perfectly in antiphase with relative vorticity, so this term is the only term able to dissipate relative vorticity from area $M_{1}$. 


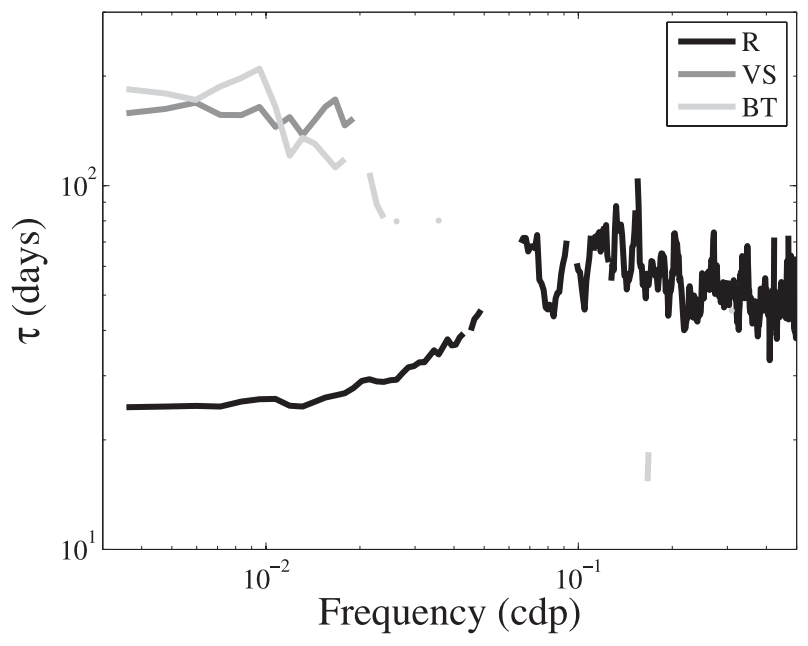

FIG. 9. Decay time scale $\tau$ as estimated from Eq. (11) for the terms $R$, VS, and BT. Values are plotted only where the coherence between the forcing and the cospectrum exceeds the $95 \%$ significance level.

A different balance can be observed for a small frequency range between $1 / 20$ and $1 / 12 \mathrm{cpd}$. In this band VS loses some of its coherence with $Z$ (and $F$ ), while BT and $Z$ are now marginally coherent and approximately in quadrature. The fact that $\mathrm{BT}$ and $d Z / d t$ are in phase suggests that Rossby wave dynamics play a role here. These waves represent a local exchange between relative and potential vorticity, but do not remove total vorticity from the area. Again, friction $R$ is the only process capable of dissipating vorticity.

For frequencies lower than about $1 / 20 \mathrm{cpd}, R$ takes over as the dominant term in the residual, increasing its power toward lower frequencies, and becoming the dominant term (after forcing) for the lowest frequencies. The balances for the modal area $M_{2}$ show a picture that is fully consistent with that of $M_{1}$.

To see what decay time scales are implied by the different terms in the vorticity equation, we estimate $\tau$ from the phase relations between $Z$ and the different terms of the vorticity equation (Fig. 9). Here we base our estimate on Eq. (11), using only the cospectrum. It is clear that friction is the only process capable of removing vorticity from the modal areas. For low frequencies, the associated damping time scale is of order 25 days. This is consistent with the decay rates established from the normal-mode analysis, and with the decay rates estimated from the coherence between wind forcing and the projection time series of $u$ and $v$ from the model.

The analysis supports the conclusion by Koblinsky (1990) that a Sverdrup regime cannot be sustained in a region where contours of $f / H$ are closed, as the con-

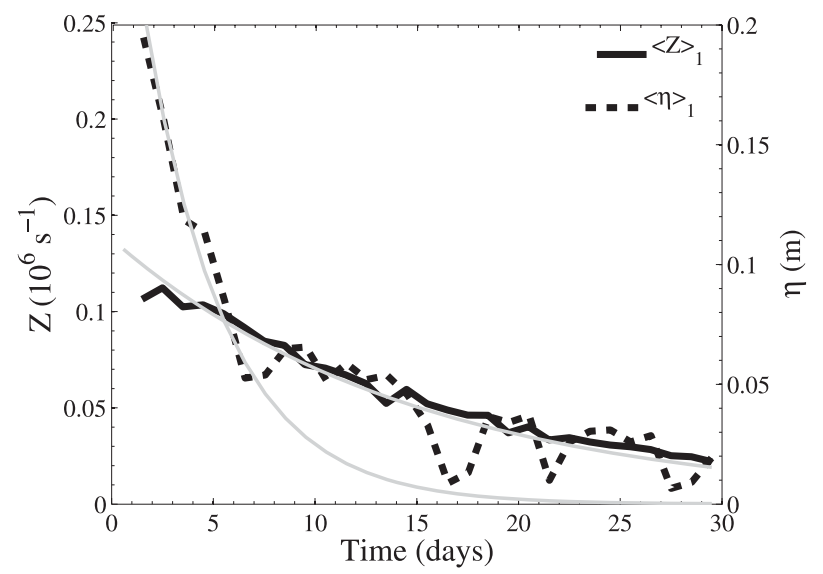

FIG. 10. SSH (dashed line) and barotropic relative vorticity (solid line), averaged over the modal area $M_{1}$, for an integration initialized with a patch of random values [taken from a uniform distribution $U(0,10)$ ] for $\eta$ in $M_{1}$. Gray lines denote exponential decay with 4- and 15-day time scales.

straints imposed by mass conservation prevent net advection across contours of $f / H$. However, Rossby wave dynamics do seem to play a role in the modal areas for both the high- and intermediate-frequency ranges.

\section{c. The role of Rossby waves}

The vorticity analysis does not provide an answer as to why SSH in the modal areas decays so rapidly, despite the slow frictional decay of the actual modes. To study this in more detail, we performed additional experiments in which we initialized the modal areas by a random perturbation of sea surface height $\eta$. The values are taken from a uniform distribution $U(0,10)$, so the average value of SSH in the modal areas is $5 \mathrm{~m}$ (for a linear system the actual amplitude of the perturbation does not matter for the conclusions). The subsequent evolution of $\eta$ and $Z$ for the run initialized in area $M_{1}$ shows very interesting behavior (Fig. 10). The decay of relative vorticity is clearly exponential with a long time scale, consistent with the vorticity analysis of the previous section. The decay of $\eta$, on the other hand, displays two distinct phases. The first phase, lasting for about a week, sees $\eta$ decaying on a very fast $\mathcal{O}$ (4 days) time scale, while subsequent adjustment follows the slow decay dictated by $Z$.

This state of affairs suggests that an initial perturbation in SSH, and the geostrophic flow that remains after the initial geostrophic adjustment has taken place, have only a small projection on the barotropic mode. This component of the flow is exactly balanced and decays slowly on the frictional time scale. The remaining component is unbalanced, however, and disintegrates rapidly. In view of the time scale, the most likely candidates 
a)

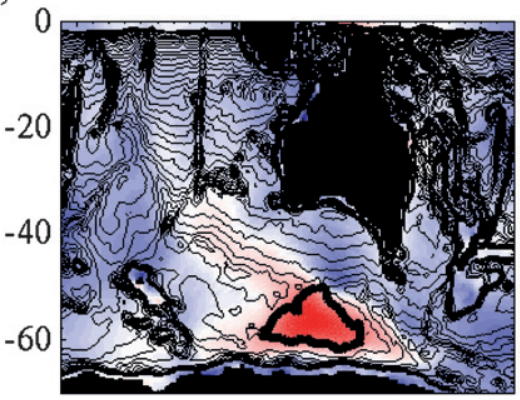

c)

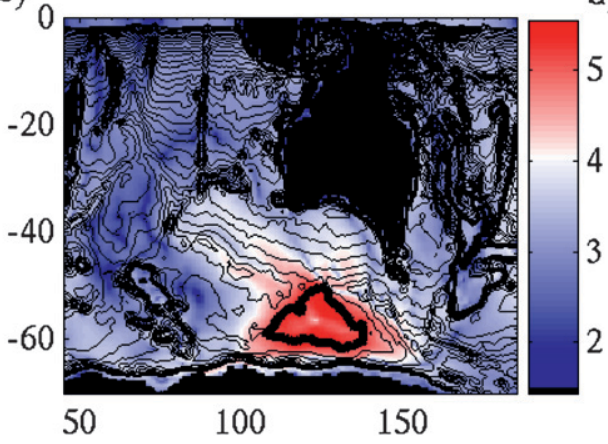

b)
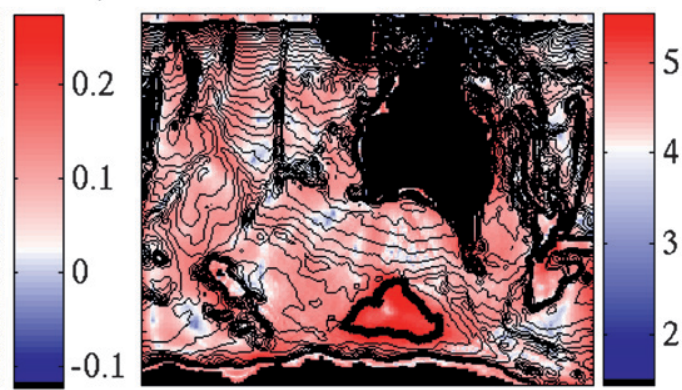

d)

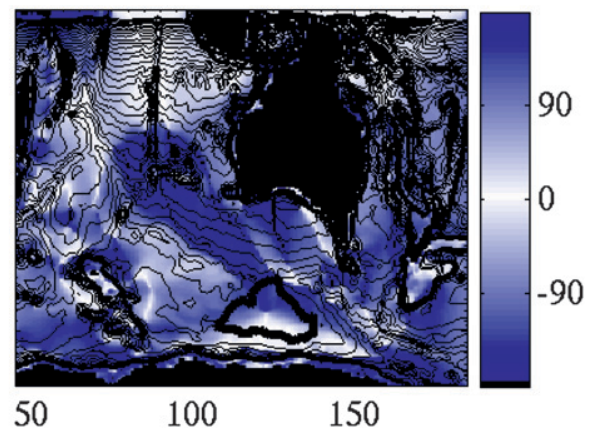

FIG. 11. Energy redistribution during day 2 of the transient run initialized with a patch of random SSH values in the modal area $M_{1}$ : (a) $\bar{\eta}$, where the overline denotes integration over day 2; (b) base-10 logarithm of the amplitude of the energy flux, defined as $\overline{H \mathbf{u} \eta}$; (c) as in (b), but for the energy flux brought about by the low-frequency motions, defined as $F_{\mathrm{LF}}=\overline{H \tilde{\mathbf{u}} \tilde{\eta}}$, where the tildes indicate variables averaged over 13-h windows; and (d) direction (in degrees with respect to due east) of $F_{\mathrm{LF}}$. Thick black contours denote the area $M_{1}$ of initialized SSH, whereas thin contours denote isolines of $f / H$.

for the rapid adjustment are Rossby waves. It is important to emphasize here that Rossby waves transport energy, but not vorticity. Linear Rossby wave dynamics are fundamentally based on the conservation of total vorticity, through the local and temporary exchange of relative and potential vorticity [the left-hand-side terms in Eq. (13)]. So although Rossby waves transport energy away from the initialized patches, the vorticity in the modal areas is left behind and is subject to frictional decay.

To test the conclusions by WD02 and Vivier et al. (2005) that energy leaks away through topographically trapped Rossby waves, we consider the propagation of anomalies and energy in more detail (Fig. 11). Even a full day after initialization, the total energy flux (defined as $\mathbf{F}=\overline{H \mathbf{u} \eta}$, where the overline indicates averaging over day 2) is dominated by inertia-gravity waves. The amplitude pattern (Fig. 11b) seems random, and does not reveal an obvious correlation with bathymetry. However, the energy flux brought about by subinertial motions (variables filtered with a boxcar window of $13 \mathrm{~h}$ ) does display a few preferred directions of energy propagation. Although the strongest energy fluxes are found in the area $M_{1}$, where the barotropic mode re- circulates energy in an anticyclonic direction, the Southeast Indian Ridge clearly serves as a corridor for the propagation of SSH anomalies (Fig. 11a) and energy of the low-frequency flow (Figs. 11c,d). Other directions are eastward along the southeastern section of the Southeast Indian Ridge, and westward into the western AAB.

\section{The origin of high SSH variance in the $\mathbf{A A B}$}

\section{a. Reconstructing the modal contribution}

In this section the modal excitation is put in the context of general SSH variability in the AAB region. To this end, we revisit the forced-model integration as discussed in section 3a. If we assume that the modal amplitude is reflected by the projection time series of $u$ and $v$ (as discussed in that section), we can construct an estimate of the modal amplitude time series by taking the simple average $\gamma_{i} \approx\left(\gamma_{i}^{u}+\gamma_{i}^{v}\right) / 2$. These time series now allow us to reconstruct the contribution of the modes to the dynamical fields according to Eq. (2), and to determine the residual $\mathbf{S}_{r}=\mathbf{S}-\mathbf{S}_{m}$.

Figure 12 shows the contribution of the two dominant modes to the SSH variability in the AAB area of the 

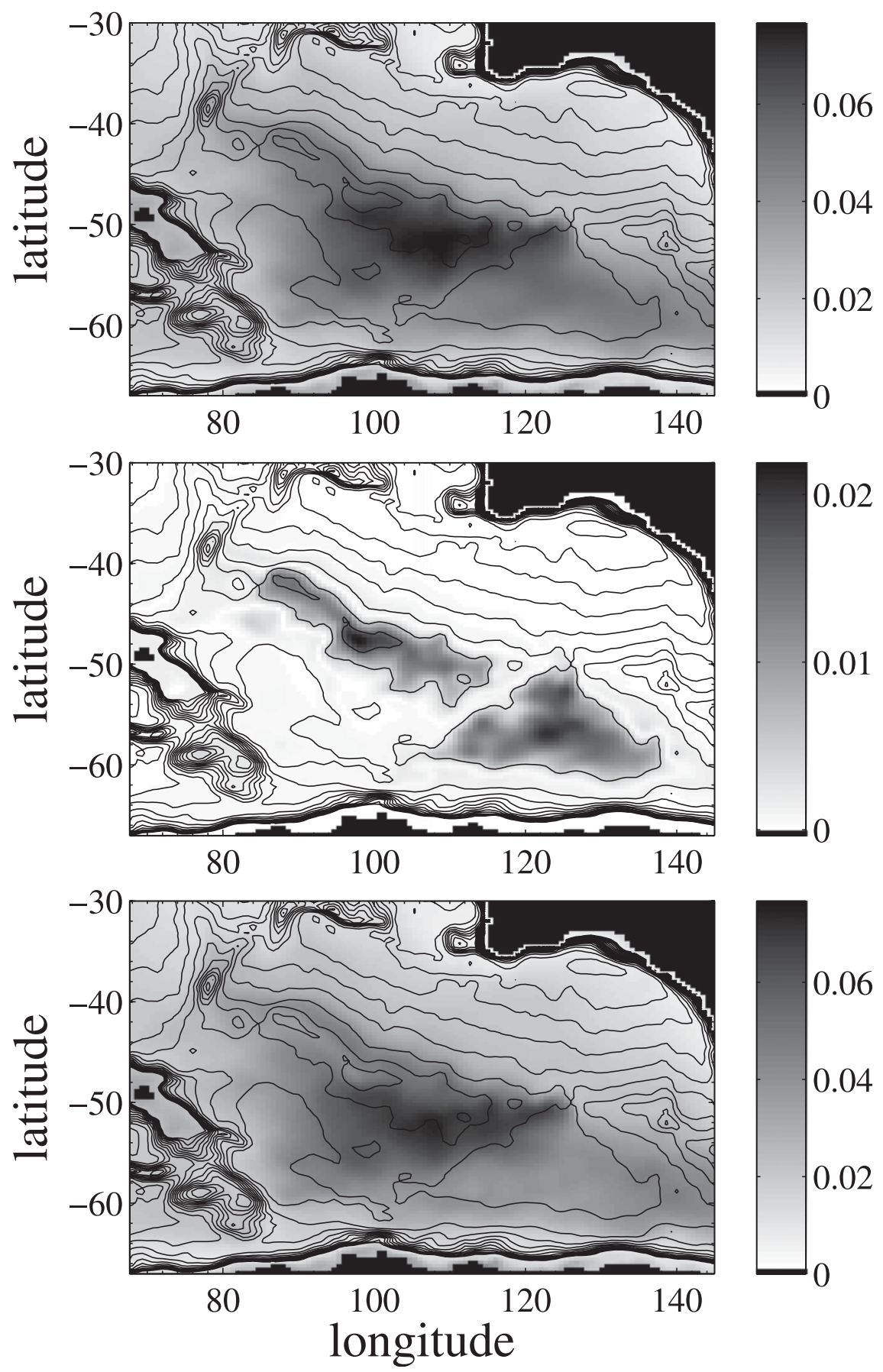

FIG. 12. SSH variability for the forced-model integration. Standard deviation of (top) the full SSH field, $S^{\eta}$; (middle) the contributions of modes 1 and 2, $S_{m}^{\eta}$; and (bottom) the residual, $S_{r}^{\eta}=$ $S^{\eta}-S_{m}^{\eta}$

forced-model integration. It is clear that the contribution of the truly free modes is relatively small. Averaged over the modal areas $M_{1}$ and $M_{2}$, the modes account for approximately $20 \%$ of the standard deviation of SSH variability, and $10 \%$ of the vertically integrated kinetic energy.
The pattern of SSH variability in Fig. 12a shows obvious resemblances to the dominant (rotated) EOF in Fig. 1. Although clearly tied to the specific distribution of $f / H$, the pattern of enhanced variability cannot be explained by the excitation of truly free modes alone. 

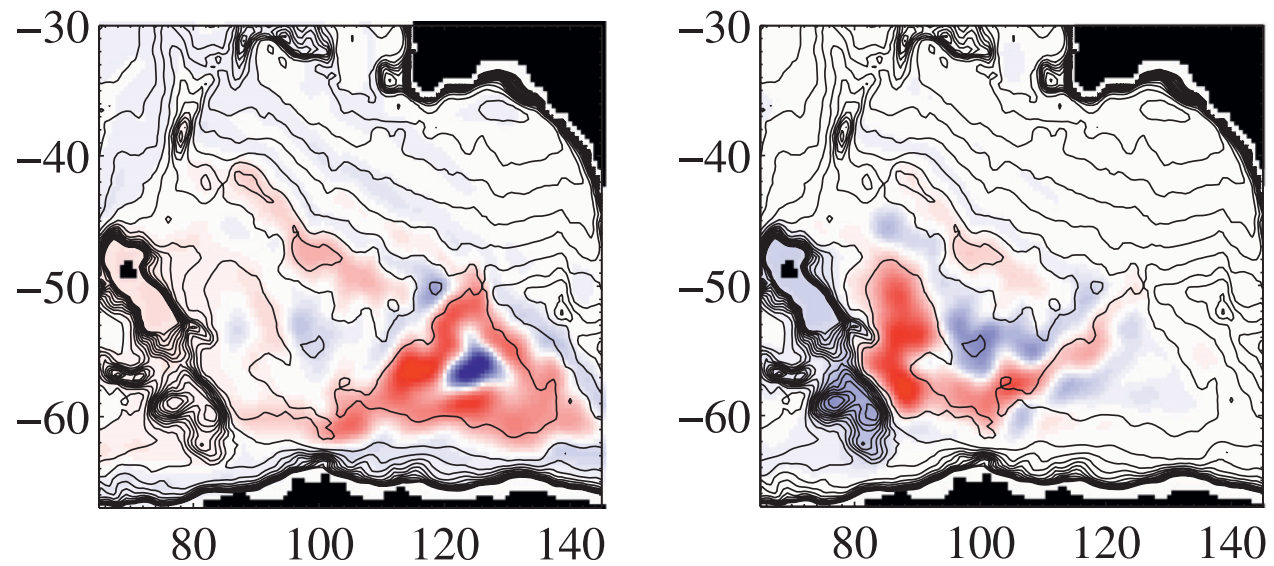

FIG. 13. SSH of two additional modes found in the AAB. Decay time scales are 12.8 and 12.0 days for the standard parameter values used here.

\section{b. Other free modes}

Although modes 1 and 2 are the least-damped modes found in the area (i.e., they have the smallest decay rates), they are not the only modes that could play a role in the variability of the AAB. Figure 13 shows two (stationary) modes that have substantially larger decay rates than modes 1 and 2 . Still, the mode pictured in the right-hand panel is located in the western $\mathrm{AAB}$, and might be responsible for the variability reflected by rotated EOF 2 in Fig. 1. These modes can be excited if the forcing projects sufficiently on their spatial patterns.

Although deeper in the spectrum and hence more damped than the dominant modes 1 and 2 , these modes are still likely to decay on biweekly time scales, for appropriate values of friction. An $\mathcal{O}(4$ days) decay time scale of the SSH variability would rely on the same mechanism as described for mode 1 in section $3 \mathrm{c}$. Hence, the signature of the actual, slow, modal decay would again be hidden by the rapid disintegration of the unbalanced flow components.

\section{c. Almost free modes}

Another possibility is that almost free modes exist in the areas of the AAB where contours of $f / H$ are almost closed. This concept was invoked by Hughes et al. (1999) to explain high levels of variability around the Antarctic continent. There, contours of $f / H$ are almost continuous around the continent, except for a few bottlenecks like the Drake Passage. A quasi-modal response can be generated along these contours if an appropriate wind stress curl is available to push the flow over the obstructing bathymetry.

The thick white and yellow lines in Fig. 14 denote two contours of $f / H$ that encircle most of the AAB. It is clear that an almost free flow along these contours could be sustained if it received an occasional push to switch contours at only two locations, north of the Kerguelen Plateau and north of the eastern AAB. Such a flow would be associated with a coherent change in $\mathrm{SSH}$ in the entire area encompassed by the contours. Indeed, Fig. 14 shows that $\mathrm{SSH}$ in the entire region of the AAB is significantly correlated to the dominant principal component, derived from an EOF analysis of the model SSH data (significant negative correlations outside the region reflect far-field response due to mass conservation). Meredith and Hughes (2004) found a similar distribution of significant correlations with altimeter-derived $\mathrm{SSH}$ anomalies at the location of Amsterdam Island $\left(37^{\circ} 49^{\prime} \mathrm{S}, 77^{\circ} 33^{\prime} \mathrm{E}\right)$.

Intriguingly, Weijer and Gille (2005) showed that a characteristic decay time scale of the southern mode is about 4 days. This decay was found to be related to form stress experienced at the constricting areas. If a similar process would dampen an almost free mode in the AAB on a comparable time scale, then the excitation and decay of such a mode would be consistent with the rapid decay of SSH observed in the modal areas.

\section{d. Trapping of transient signals}

A final contribution to the enhanced SSH variability in the $\mathrm{AAB}$ region is suggested by inspection of the time evolution of the SSH field (not shown): anomalies of $\mathrm{SSH}$ seem to travel preferably along contours of $f / H$. If the direction of the energy flux associated with the waves is related to the propagation direction of the waves, then the flux of energy across contours of $f / H$ should be substantially weaker than the flux along those contours. Consequently, energy deposited within an area of (almost) closed $f / H$ contours is trapped, leading to enhanced SSH variability within that area. 


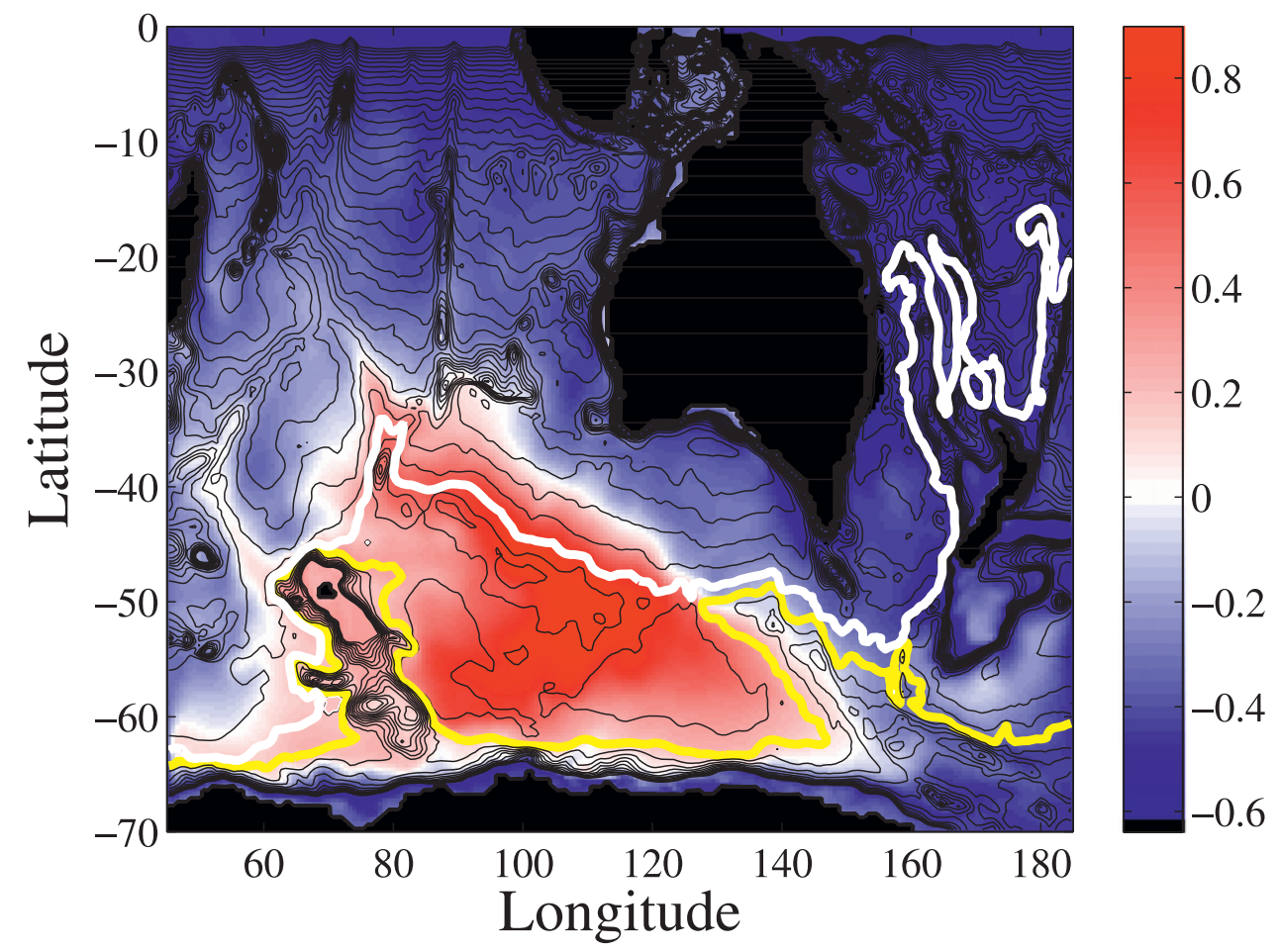

FIG. 14. Correlation between SSH and the first principal component of an EOF analysis of SSH fields of the forced-model integration. Correlations exceeding \pm 0.003 are significantly different from zero. White and yellow contours denote the isolines $f / H=2.80 \times 10^{-8} \mathrm{~m}^{-1} \mathrm{~s}^{-1}$ and $3.16 \times 10^{-8} \mathrm{~m}^{-1} \mathrm{~s}^{-1}$.

To test whether energy fluxes indeed tend to align with contours of $f / H$, we calculated the angle between the gradient of $f / H$ and the time-averaged energy fluxes, defined here, as before, as $\mathbf{F}=\overline{H \mathbf{u} \eta}$. For this calculation, $f / H$ was smoothed an arbitrary 4 times using a 1-4-1 filter, to emphasize the larger scales of bathymetry that matter to the propagation of barotropic waves. Figure 15 confirms that the predominant direction of energy fluxes is along contours of potential vorticity, with $74 \%$ of data points in the plotted area having angles between $\pi / 4$ and $3 \pi / 4$ relative to the gradient of $f / H$. Clearly, the convoluted nature of $f / H$ contours in the $\mathrm{AAB}$ makes it challenging for energy to find pathways to escape the region.

The importance of the trapping of energy by the bathymetry of the eastern $\mathrm{AAB}$ is illustrated by a calculation similar to the one described in section 3c, namely, an unforced run initialized with a patch of random SSH in area $M_{1}$, but now with a flat bottom. The initial decay of SSH averaged over the initialized area now takes place on a time scale of just 2 days (instead of 4 days). This suggests that the specifics of the bathymetry in the $\mathrm{AAB}$ indeed slow down the removal of energy from, at least, modal area $M_{1}$. In addition, SSH went through zero during day 5 (instead of remaining positive for the entire simulation), since obviously no mode was excited in the flat-bottom case.

\section{Summary and discussion}

A mode is a special circulation pattern whose structure remains spatially coherent when excited. The modes presented here represent flows along closed contours of $f / H$, which makes them neutral to a common source/sink of relative vorticity: flow across contours of $\mathrm{f} / \mathrm{H}$. Once such a circulation pattern is established, it can only lose vorticity (and energy) through frictional effects. In general, an arbitrary flow distribution has a nonzero projection on the modal patterns, so it is reasonable to assume that modes are continuously being excited by wind forcing. However, the projection may be small, so that their dynamics are hard to discern among the rapid disintegration of the flow components that are not in balance. This point is illustrated in Fig. 10, which shows that it can take a week for the unbalanced components to die out, and for the decay of the balanced flow to be revealed.

In this paper we have studied the role of topographically trapped barotropic modes in generating intraseasonal variability in the AAB. Using a shallow-water model we found two major barotropic normal modes in the AAB. Both modes are trapped by closed contours of $f / H$ : mode 1 is confined to the abyssal plain of the eastern $\mathrm{AAB}$, while mode 2 is constrained on the top of the 


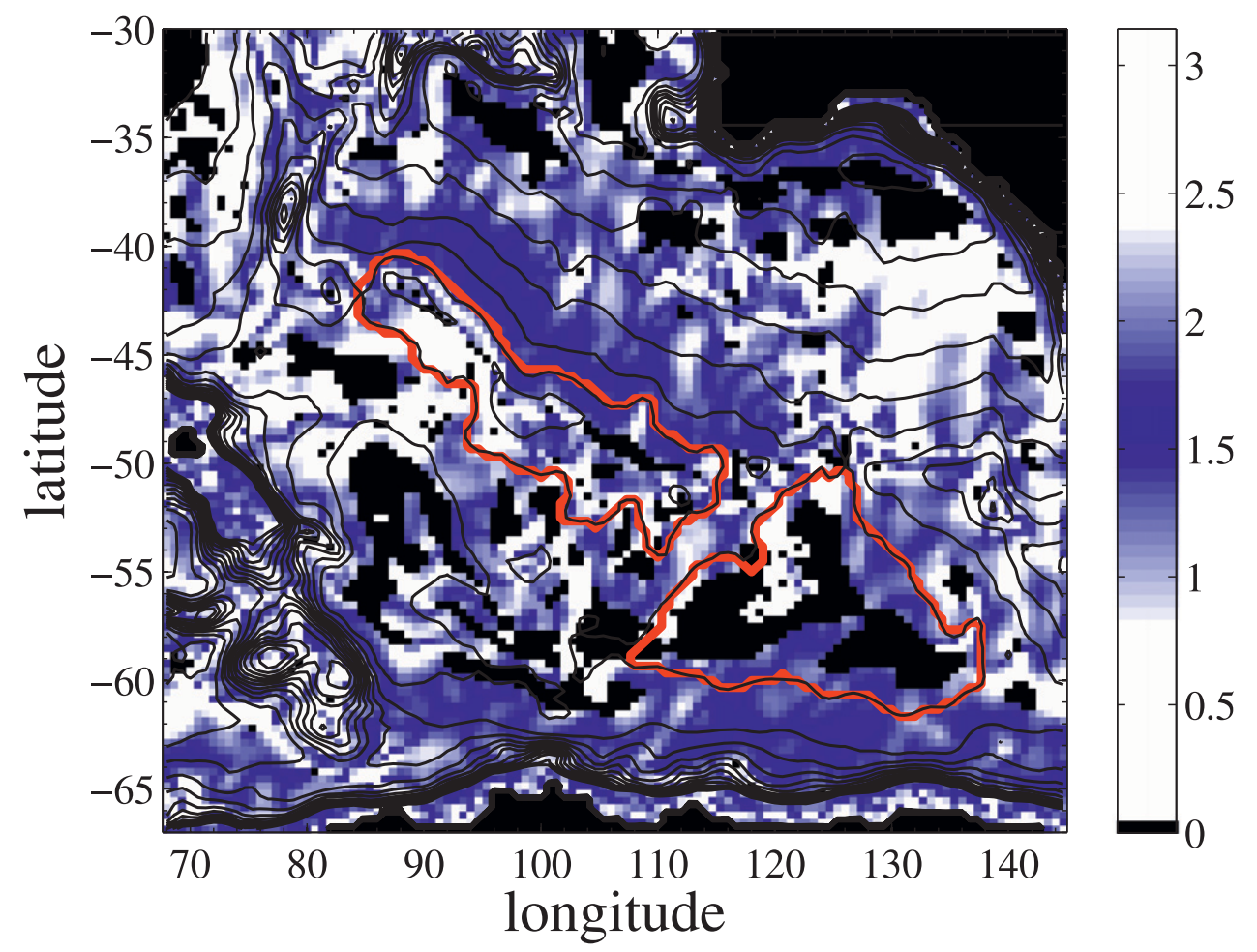

FIG. 15. Angle (in radians) between time-averaged energy flux and the gradient of $f / H$. Flat areas where $|\nabla(f / H)|<\epsilon$ are indicated in black, where $\epsilon=6 \times 10^{-15}$ is an arbitrarily chosen cutoff value.

Southeast Indian Ridge. Both modes are stationary and do not oscillate when experiencing exponential decay.

That these modes indeed account for a part of the variance was confirmed by a forward integration of the SW model. Projection of the velocity patterns associated with each mode onto the model output resulted in time series that were found to be significantly coherent with wind stress curl, averaged over the modal areas. The long $\mathcal{O}$ (20 days) phase lag between forcing and response was found to be consistent with frictional spindown of the barotropic modes. Indeed, a detailed analysis of the vorticity balance showed that friction is the only process capable of removing vorticity from the modal areas.

In contrast, it was found that SSH decays rapidly in the modal areas, in agreement with the results of WD02. This rapid $\mathcal{O}$ (4 days) decay seems at odds with the picture of slow, frictional spindown. However, additional experiments suggested that this decay reflects the adjustment of the unbalanced part of the flow field through the emission of Rossby waves. A crucial aspect of this adjustment process is that (linear) Rossby waves transport energy away from the modal areas (hence the rapid drop in sea level), but not vorticity (hence the slow decay of relative vorticity).

The barotropic modes were found to account for only $20 \%$ of the standard deviation of SSH in the modal areas, and for about $10 \%$ of the kinetic energy. Despite the visual resemblance between the barotropic modes and the dominant EOFs, other mechanisms have to be responsible for the remaining part of the variability. Candidates that rely equally strongly on the particular distribution of potential vorticity in the $\mathrm{AAB}$ are (i) other free modes that, despite having larger decay rates than modes 1 and 2, could be excited if the spatial pattern of the forcing is favorable; (ii) almost free modes in areas that are almost fully enclosed by contours of $f / H$, requiring only an occasional push over a bathymetric obstacle; and (iii) propagating signals that are trapped by the closed or almost closed contours of $f / H$. These processes will be the subject of further study.

This study has shed some light on the excitation and decay of topographically trapped modes in the AAB. In principle, the excitation of barotropic modes is a relatively simple process, and once their spatial patterns are known, they should be easily recognizable in observations. In addition, observations of modal decay could help constrain estimates of bottom friction and eddy viscosity in the Southern Ocean (see Fig. 4). However, the results of this study show that the excitation of the truly free modes in the $\mathrm{AAB}$ is relatively weak, and that their SSH expressions appear to be overwhelmed by more energetic adjustment processes. 
Acknowledgments. This research was supported by NSF through Grant 0424703, and by the Climate Change Prediction Program of the U.S. Department of Energy Office of Science (WW). Los Alamos National Laboratory is operated by the Los Alamos National Security, LLC for the National Nuclear Security Administration of the U.S. Department of Energy under Contract DEAC52-06NA25396. STG was supported by the National Aeronautics and Space Administration under JPL Contract 1224031. FV gratefully acknowledges CNES and CNRS for their support. Wind stress curl data were provided by the Data Support Section of the Computational and Information Systems Laboratory at the National Center for Atmospheric Research. NCAR is supported by grants from the National Science Foundation. The altimeter products were produced by Ssalto/ Duacs and distributed by AVISO, with support from CNES. We thank two anonymous reviewers for constructive comments, and Dr. L.-L. Fu (JPL) for generously making his data available to us.

\section{APPENDIX}

\section{The Shallow-Water Model}

\section{a. Model equations}

The modal response of the Australia-Antarctic Basin is studied using a barotropic shallow-water model. The set of equations is given by

$$
\begin{aligned}
\frac{\partial u}{\partial t}+ & \frac{u}{r_{0} \cos \phi} \frac{\partial u}{\partial \lambda}+\frac{v}{r_{0}} \frac{\partial u}{\partial \phi}-\frac{u v}{r_{0}} \tan \phi-2 \Omega v \sin \phi \\
= & -\frac{g}{r_{0} \cos \phi} \frac{\partial \eta}{\partial \lambda}+A_{h}\left(\nabla^{2} u-\frac{u}{r_{0}^{2} \cos ^{2} \phi}-\frac{2 \sin \phi}{r_{0}^{2} \cos ^{2} \phi} \frac{\partial v}{\partial \lambda}\right) \\
& +\frac{\tau^{\lambda}}{\rho_{0} h}-r u, \\
\frac{\partial v}{\partial t}+ & \frac{u}{r_{0} \cos \phi} \frac{\partial v}{\partial \lambda}+\frac{v}{r_{0}} \frac{\partial v}{\partial \phi}+\frac{u^{2}}{r_{0}} \tan \phi+2 \Omega u \sin \phi \\
= & -\frac{g}{r_{0}} \frac{\partial \eta}{\partial \phi}+A_{h}\left(\nabla^{2} v-\frac{v}{r_{0}^{2} \cos ^{2} \phi}+\frac{2 \sin \phi}{r_{0}^{2} \cos ^{2} \phi} \frac{\partial u}{\partial \lambda}\right) \\
& +\frac{\tau^{\phi}}{\rho_{0} h}-r v, \quad \text { and } \\
& \frac{\partial \eta}{\partial t}+\frac{1}{r_{0} \cos \phi}\left[\frac{\partial(h u)}{\partial \lambda}+\frac{\partial(h v \cos \phi)}{\partial \phi}\right]=0
\end{aligned}
$$

Here, $(u, v)$ are the depth-averaged zonal and meridional velocity components; $h=H_{0}+\eta-h_{b}$ is total water depth, with $H_{0}$ maximum depth, $\eta$ sea surface elevation, and $h_{b}$ bathymetry; $\lambda$ and $\phi$ are east longitude and lati-
TABLE A1. Model parameters and their standard values.

\begin{tabular}{lll}
\hline \hline Symbol & \multicolumn{1}{c}{ Scales } & \multicolumn{1}{c}{ Value } \\
\hline$H_{0}$ & Maximum depth & $7328 \mathrm{~m}$ \\
$r_{0}$ & Earth's radius & $6.37 \times 10^{6} \mathrm{~m}$ \\
$\Omega$ & Earth's rotation rate & $7.272 \times 10^{-5} \mathrm{~s}^{-1}$ \\
$G$ & Gravitational constant & $9.8 \mathrm{~m} \mathrm{~s}^{-2}$ \\
$\rho$ & Density & $1020 \mathrm{~kg} \mathrm{~m}^{-3}$ \\
$A_{h}$ & Horizontal viscosity & $3 \times 10^{3} \mathrm{~m}^{2} \mathrm{~s}^{-1}$ \\
$r$ & Bottom friction & $2 \times 10^{-7} \mathrm{~s}^{-1}$ \\
\hline
\end{tabular}

tude, respectively, expressed in radians; and $\tau^{\lambda}$ and $\tau^{\phi}$ denote zonal and meridional wind stress. Model parameters and their values are tabulated in Table A1.

\section{b. Normal-mode analysis}

The discretized SW equations can be expressed as

$$
\mathbf{M} \frac{\partial \mathbf{x}}{\partial t}+\mathbf{L x}+\mathbf{N}(\mathbf{x})=0
$$

where $\mathbf{x}$ denotes the state vector, $\mathbf{M}$ the mass matrix, $\mathbf{L}$ the linear operators, and $\mathbf{N}$ the nonlinear operators. Let $\overline{\mathbf{x}}$ be a steady solution of this system, and let $\tilde{\mathbf{x}}$ be a small perturbation such that $\mathbf{x}=\overline{\mathbf{x}}+\tilde{\mathbf{x}}$. Neglecting terms quadratic in the perturbations, we get

$$
\mathbf{M} \frac{\partial \tilde{\mathbf{x}}}{\partial t}+\mathbf{L} \tilde{\mathbf{x}}+\mathbf{N}_{x}(\overline{\mathbf{x}}) \tilde{\mathbf{x}}=0
$$

where $\mathbf{L}+\mathbf{N}_{x} \equiv \mathbf{A}$ is the Jacobian matrix. Inserting the generic form $\tilde{\mathbf{x}}=\hat{\mathbf{x}} e^{\sigma t}$ leads to a generalized eigenvalue problem of the form

$$
\mathbf{A} \hat{\mathbf{x}}=\sigma \mathbf{B} \hat{\mathbf{x}}
$$

where $\mathbf{B}=-\mathbf{M}$. Here, $\sigma=\sigma_{r}+i \sigma_{i}$ is the complex growth factor: the decay time scale of $\tilde{\mathbf{x}}$ (and hence the linear stability of the state $\overline{\mathbf{x}}$ ) is determined by its real part according to $1 / \sigma_{r}$, while the oscillation frequency is given by $2 \pi / \sigma_{i}$. The eigenvectors $\hat{\mathbf{x}}$ for each $\sigma$ are called the eigenmodes associated with $\overline{\mathbf{x}}$. If $\sigma_{i} \neq 0$ the mode is oscillatory and these eigenmodes are complex: $\hat{\mathbf{x}}=$ $\hat{\mathbf{x}}_{r}+i \hat{\mathbf{x}}_{i}$. The oscillation proceeds as $\hat{\mathbf{x}}_{r} \rightarrow-\hat{\mathbf{x}}_{i} \rightarrow-\hat{\mathbf{x}}_{r} \rightarrow$ $\hat{\mathbf{x}}_{i} \rightarrow \cdots$.

For this study, a motionless background solution $\overline{\mathbf{x}}$ is considered. This assumption renders $\mathbf{N}_{x}(\overline{\mathbf{x}})=0$ and basically eliminates all nonlinear advective terms, as well as nonlinear inertial terms that arise from the spherical geometry.

A powerful method to solve the generalized eigenvalue problem (A6) is the Jacobi-Davidson QZ method (JDQZ; Sleijpen and Van der Vorst 1996). This method calculates eigenvalues that are closest to a prespecified 
target value. Once an eigenvalue has been found, and the corresponding eigenvector has been determined, one can easily trace the evolution of this eigenmode versus model parameters.

\section{REFERENCES}

Chao, Y., and L.-L. Fu, 1995: A comparison between the TOPEX/ POSEIDON data and a global ocean general circulation model during 1992-1993. J. Geophys. Res., 100, 24965 24976.

Ducet, N., P. Y. L. Traon, and G. Reverdin, 2000: Global highresolution mapping of ocean circulation from TOPEX/ Poseidon and ERS-1 and -2. J. Geophys. Res., 105, 19 47719498.

Fu, L.-L., 2003: Wind-forced intraseasonal sea level variability of the extratropical oceans. J. Phys. Oceanogr., 33, 436-449.

— lite altimetry and high-resolution computer simulation. Bull. Amer. Meteor. Soc., 77, 2625-2636.

Fukumori, I., R. Raghunath, and L.-L. Fu, 1998: Nature of global large-scale sea level variability in relation to atmospheric forcing: A modeling study. J. Geophys. Res., 103, 5493-5512.

Hannan, E. J., 1970: Multiple Time Series. Wiley, 536 pp.

Hughes, C. W., M. P. Meredith, and K. J. Heywood, 1999: Winddriven transport fluctuations through Drake Passage: A southern mode. J. Phys. Oceanogr., 29, 1971-1992.

Koblinsky, C. J., 1990: The global distribution of $f / H$ and the barotropic response of the ocean. J. Geophys. Res., 95, 32133218.

Meredith, M. P., and C. W. Hughes, 2004: On the wind-forcing of bottom pressure variability at Amsterdam and Kerguelen Islands, southern Indian Ocean. J. Geophys. Res., 109, C03012, doi:10.1029/2003JC002060.

Milliff, R. F., J. Morzel, D. B. Chelton, and M. H. Freilich, 2004: Wind stress curl and wind stress divergence biases from rain effects on QSCAT surface wind retrievals. J. Atmos. Oceanic Technol., 21, 1216-1231.

Parks, T. W., and J. H. McClellan, 1972: Chebyshev approximation for nonrecursive digital filters with linear phase. IEEE Trans. Circuit Theory, CT-19, 189-194.

Ponte, R. M., and P. Gaspar, 1999: Regional analysis of the inverted barometer effect over the global ocean using TOPEX/ POSEIDON data and model results. J. Geophys. Res., 104, 15 587-15 601.

Richman, M. B., 1986: Rotation of principal components. J. Climatol., 6, 293-335.

Sleijpen, G. L. G., and H. A. Van der Vorst, 1996: A JacobiDavidson iteration method for linear eigenvalue problems. SIAM J. Matrix Anal. Appl., 17, 410-425.

Vivier, F., K. A. Kelly, and M. Harismendy, 2005: Causes of largescale sea level variations in the Southern Ocean: Analyses of sea level and a barotropic model. J. Geophys. Res., 110, C09014, doi:10.1029/2004JC002773.

Webb, D. J., and B. A. De Cuevas, 2002a: An ocean resonance in the Indian sector of the Southern Ocean. Geophys. Res. Lett., 29, 1664, doi:10.1029/2002GL015270.

$\longrightarrow$, and 2002b: An ocean resonance in the Southeast Pacific. Geophys. Res. Lett., 29, 1252, doi:10.1029/2001GL014259.

— ability in the southeast Pacific Ocean. J. Phys. Oceanogr., 33, 1044-1056.

Weijer, W., 2008: Normal modes of the Mascarene Basin. Deep-Sea Res. II, 55, 128-136.

— , and S. T. Gille, 2005: Adjustment of the Southern Ocean to wind forcing on synoptic time scales. J. Phys. Oceanogr., 35, 2076-2089.

— F. Fivier, S. T. Gille, and H. Dijkstra, 2007a: Multiple oscillatory modes of the Argentine Basin. Part I: Statistical analysis. J. Phys. Oceanogr., 37, 2855-2868.

,,,--- and,$- 2007 \mathrm{~b}$ : Multiple oscillatory modes of the Argentine Basin. Part II: The spectral origin of basin modes. J. Phys. Oceanogr., 37, 2869-2881. 